Revista de Psicología Vol. 36 (2), 2018 (ISSN 0254-9247)

\title{
Perfil neuropsicológico de atención y memoria en víctimas del conflicto armado colombiano ${ }^{1}$
}

\author{
José Alberto Luna Hernández², Paola Daniela Rodríguez Rojas ${ }^{3}$, \\ Isabel Hernández Arteaga ${ }^{4}$ \\ Universidad Mariana, Colombia ${ }^{2,3}$, Universidad Cooperativa de Colombia ${ }^{4}$
}

La investigación tuvo como objetivo determinar el rendimiento neuropsicológico de atención y memoria en víctimas de desplazamiento forzado a causa del conflicto armado colombiano. Se utilizó una metodología de tipo descriptivo comparativo, con un paradigma cuantitativo. Como muestra fueron tomadas 20 personas distribuidas en dos grupos: A (9 personas con TEPT) y B (11 personas sin TEPT), quienes fueron evaluadas con la batería NEUROPSI (atención y memoria). Los resultados indican que el grupo con TEPT tiene un rendimiento inferior en comparación al grupo sin TEPT en el proceso atencional, mientras que a nivel de memoria el grupo con TEPT manifiesta una mejor ejecución de las tareas; siendo así, se indica que las dificultades existentes en atención en el grupo A, pueden relacionarse con la presencia del TEPT como una variable influyente en la aparición de déficits cognitivos.

Palabras clave: neuropsicología, atención, memoria, conflicto armado

\section{Attention and Memory Neuropsychological profile in the Colombian armed}

This study sought to determine the neuropsychological performance of attention and memory in victims of forced displacement due to the Colombian armed conflict. The methodology used was of comparative descriptive type, with a quantitative paradigm.

1 Investigación aprobada y financiada por la Universidad Mariana, a través del Centro de Investigaciones, Programa de Psicología.

2 Magíster en Neuropsicología Clínica, especialista en Evaluación y Diagnóstico Neuropsicológico y docente de Maestría en Salud Mental del CES- Medellín y coordinador del Laboratorio de Psicología de la Universidad Mariana. Dirección Postal: Condominio Aquine 3 sector 1 casa c-3 Pasto, Nariño, Colombia. Contacto: jluna@umariana.edu.com

3 Egresada de Psicología de la Universidad Mariana. Dirección postal: Carrera 29 N³ Oeste 23 Pasto, Narińo, Colombia. Contacto: paoladrodriguez@umariana.edu.com

4 Posdoctora en Investigación Cualitativa, docente de la Escuela de Posgrados en Educación en la Universidad Cooperativa de Colombia, investigadora del Centro de Investigaciones en Docencia Universitaria (CIDU). Dirección postal: Carrera 6a_No 11-51. La Candelaria Bogotá, Colombia. Contacto: Isabel.hernandez@ucc.edu.com 
The sample was of 20 people distributed in group A (9 people with PTSD) and group B (11 people without PTSD). Participants were evaluated with NEUROPSI battery (attention and memory). Results show that the group with PTSD had a lower performance compared to the group without PTSD in attention, while in memory the group with PTSD showed a better performance of the tasks; the existing difficulties in group A can be related to PTSD as an influential variable in the appearance of cognitive deficits.

Keywords: neuropsychology, attention, memory, armed conflict.

\section{Perfil neuropsicológico da atençáo e memória em vítimas do conflito armado colombiano}

A pesquisa foi como objetivo para determinar o rendimento neuropsicológico da atençáo e memória em vítimas do deslocamento forçado causado pelo conflito armado na Colômbia, foi utilizado uma metodologia do tipo descritivo comparativo, com um paradigma cuantitativo. Como amostra foram utilizadas 20 pessoas distribuídas no grupo A ( 9 pessoas com TEPT) e grupo B (11 pessoas sem TEPT), que foram avaliadas com a bateria NEUROPSI (atenção e memória). Os resultados indicam que o grupo com TEPT tem rendimento menor em comparação ao grupo sem TEPT em atenção, enquanto que ao nível de memória, o grupo com TEPT apresenta uma melhor execução das tarefas; sendo assim, se indica que as dificuldades existentes na atenção no grupo A, podem relacionar-se com a presença do TEPT como uma influente variável na aparição do déficit cognitivos.

Palavras chave: neuropsicologia, atenção, memória, conflito, armado.

\section{Profil neuropsychologique de l'attention et de la mémoire chez les victimes du conflit armé colombien}

L'objectif de la recherche était de déterminer la performance neuropsychologique de l'attention et de la mémoire chez les victimes de déplacements forcés dus au conflit armé colombien, une méthodologie de type descriptif comparatif a été utilisée, avec un paradigme quantitatif. L'échantillon a été prélevé 20 personnes réparties dans le groupe A ( 9 personnes atteintes du SSPT) et le groupe B (11 personnes sans ESPT), qui ont été évaluées avec la batterie NEUROPSI (attention et mémoire). Les résultats indiquent que le groupe avec TSPT a une performance inférieure par rapport au groupe sans TSPT dans le processus attentionnel, tandis qu'au niveau de la mémoire, le groupe avec TSPT montre une meilleure performance des tâches; Ainsi, il est indiqué que les difficultés existant dans l'attention dans le groupe A peuvent être liées à la présence du SSPT en tant que variable influente dans l'apparition des déficits cognitifs.

Mots-clés: neuropsychologie, attention, mémoire, conflit armé. 
El conflicto armado en Colombia con más de 50 años de trascendencia en escenarios de guerra, es un fenómeno que afectó indirectamente a toda la población del país y directamente a un número significativo de víctimas. Para Wilches (2010) al menos una de cada diez personas se desplazó de su lugar de origen, salió de su hogar y de su tierra por culpa de la guerra fruto del conflicto armado; así mismo la autora se refiere como víctima al ser humano afectado por el conflicto, por tanto, todos los colombianos pueden ser caracterizados como tal. En este sentido, el conflicto armado se constituye indudablemente en una problemática relevante en el país.

Espitia (2015) señala que la Red Nacional de Información (RNI) reportó en el año 2014 una cifra de 7.624.299 víctimas del conflicto armado en el período comprendido entre los años 1985-2014, que representa el $16 \%$ de la población total colombiana; es decir que, de cada 100 colombianos, 16 se catalogan como víctimas. Complementa el autor, que estas personas han sufrido diversos hechos de violencia, que en su mayoría apuntan a desplazamiento forzado, ya que del número de reportados previamente el $79.77 \%$ corresponden a este acontecimiento violento, por tanto, victimizante, destacando el problema fundamentalmente en "los departamentos de Antioquia (18.72\%), Bolívar (8.35\%), Magdalena (6.58\%), Choco (5.36\%), Nariño (5.28\%) y Cesar (5.24\%)" (p. 2)

Teniendo en cuenta la gran incidencia del conflicto armado colombiano según lo dimensionan los datos anteriores, autores como Hernández, Luna y Cadena (2017), suponen la destrucción y muerte como escenarios propios de la violencia en Colombia, que propician hechos perturbadores en las distintas dimensiones de la persona y la sociedad; principalmente en el desplazamiento como fenómeno de violencia que recoge la mayor cantidad de víctimas. A su vez, Jurado, Taboada, García, Denia, Mingote y Fernández (2007) expresan que las 
situaciones traumáticas a las cuales han sido expuestas estas personas generan con el paso del tiempo una serie de afectaciones o secuelas, como el Trastorno por Estrés Postraumático (TEPT), que aparece tras la vivencia de un suceso altamente amenazante o catastrófico. Asimismo, se caracteriza por la reexperimentación del evento estresante, en forma de sueños o de flash-back, hiperactivación e hiperalerta y embotamiento emocional; así como la evitación de aquellos estímulos relacionados con el evento, de forma conductual o, a menudo, en forma de amnesia postraumática.

Sin duda, a partir de estas manifestaciones sintomatológicas, se ven afectadas las diferentes esferas de la persona: psicológica, social y familiar. Pero también, según lo mencionan Calderón y Barrera (2012) se alteran de forma relevante los procesos básicos de atención y memoria, ocasionando un deterioro importante en su bienestar y calidad de vida. Complementando esta idea, Gálvez (2005) y Coelho y Costa (2010) encontraron que dificultades cognitivas asociadas a la discriminación de estímulos, así como a la retención y consolidación de información en sujetos que sufren de TEPT, se relaciona directamente con una implicación neuro-anatómica de base, sustentada por la segregación de cortisol en el organismo, comprometiendo estructuras como el hipocampo, la amígdala y la corteza prefrontal asociados a estos procesos. $\mathrm{Al}$ respecto, Hurtado (2011) señala que las investigaciones consultadas para su estudio refieren a estas estructuras como las áreas de mayor vulnerabilidad en lo tocante a este trastorno.

Por tanto, es posible sugerir una relación del padecimiento de TEPT con la aparición de déficits en procesos neuropsicológicos de atención y memoria, tal como se presenta en los resultados de investigaciones realizadas por Jurado et al. (2007), Quintero (2011), Seijas (2013) y Yaffe et al. (2010), Sin embargo, haciendo una revisión teórica del tema, existen estudios en los cuales se presentan opiniones contrarias a esta relación, pues al evaluar sujetos con y sin TEPT, se evidencia un rendimiento normal en ambos grupos, como lo presentan los resultados logrados en la investigación realizada por Neylan, Lenoci, Rothlind, Metzler, Schuff, Du, Franklin, Weiss, Weiner \& Marmar 
(2004). Considerando estos hallazgos en relación al TEPT, el presente estudio busca determinar el rendimiento neuropsicológico en atención y memoria de personas que sufrieron trastorno por estrés postraumático (TEPT) debido al desplazamiento forzado, en una de las regiones con mayor índice de violencia por el conflicto armado del país.

El presente estudio busca brindar información para una comprensión más amplia de las características que atañen a este tipo de población y de las problemáticas que surgen en torno a ser víctima del conflicto armado; en este caso, frente a la aparición de déficits cognitivos asociados al TEPT. De esta manera, se busca contribuir a que las entidades que brindan atención integral a estos grupos consideren modelos de intervención que incluyan servicios específicos a nivel neuropsicológico. Esto favorecerá los procesos de adaptación en la fase del postconflicto -por la que atraviesa actualmente el proceso de Paz en Colombia-, que perdurarán hasta que el pueblo colombiano logre vivir en una sociedad de paz (Melamed, 2014; Villarraga, 2013).

\section{Método}

Para la realización de este estudio se determinó una ruta de trabajo, a partir del paradigma de investigación cuantitativo, con enfoque empírico-analítico, de tipo descriptivo comparativo y un diseño transversal.

\section{Participantes}

La selección de los participantes se realizó contactando inicialmente un total de 30 sujetos vinculados a la Unidad de Atención y Restauración Integral a Victimas (UARIV) de Colombia, quienes pertenecen a dos asociaciones de víctimas en la Ciudad de San Juan de Pasto, capital del departamento de Nariño, Colombia. Posteriormente, por medio de cálculo estadístico y tomando como nivel de confiabilidad $(z)$ un $95 \%$ y un margen de error $(e)$ del 5\%; se determinó una muestra $(n)$ de trabajo con un total de 28 personas, como posibles participantes en el estudio. 
De esta manera fueron seleccionadas 28 víctimas del conflicto armado en Colombia, a quienes se aplicó criterios de inclusión previamente establecidos para el desarrollo del proyecto, entre estos: primero, que los sujetos sean víctimas de desplazamiento forzado causado por el conflicto armado; y segundo, que sean mayores de edad, es decir, que tengan más de 18 años (cuando se adquiere la mayoría de edad en Colombia). Al mismo tiempo, se consideró como criterio de exclusión la presencia de un diagnóstico psicológico, psiquiátrico o neurológico que impida el rastreo de TEPT. Como resultado se filtró a 20 personas, en su totalidad mujeres, con un promedio de edad que osciló en un rango entre 31 y 55 años, la mayoría de ellas con un nivel de escolaridad entre 4 y 9 ańos de estudios; con homogeneidad en cuanto al estado civil soltero, casado y unión libre. En relación a su nivel de ocupación prevalece el desempeño en actividades relacionadas al hogar, seguido del trabajo en la elaboración de artesanías. La mayoría de las participantes indica su procedencia de diferentes municipios, tanto del departamento de Narińo como del departamento del Putumayo en la zona suroccidental de Colombia, en donde el desplazamiento forzado por el conflicto armado se registra significativo (Ver tabla 1).

\section{Medición}

Durante el proceso de recolección de información se buscó comprobar la existencia de sintomatología de TEPT para la distribución de los grupos de trabajo: grupo A (con TEPT) y grupo B (sin TEPT), a partir de los cuales se contrastan los resultados encontrados. Asimismo, se determinó el rendimiento en los procesos atencionales y mnémicos entre las personas evaluadas que formaron parte del estudio.

Se comprobó la existencia de sintomatología de TEPT a través de los resultados de la Escala de Trauma Davidson (DTS) (Davidson et al., 1997) adaptado por Villafañe, Milanesio, Marcellino y Amodei (2003). La DTS es una medida de auto-aplicación que evalúa la frecuencia y gravedad de los síntomas subjetivos del TEPT. La DTS utiliza los criterios B-D que se han establecido según el DSM-IV para su diagnóstico, que hacen referencia a la reexperimentación del acontecimiento 


\section{Tabla 1}

Variables sociodemográficas de los participantes

\begin{tabular}{lcc}
\hline Variable & $\begin{array}{c}\text { Grupo A } \\
9(45 \%)\end{array}$ & $\begin{array}{c}\text { Grupo B } \\
11(55 \%)\end{array}$ \\
\hline Edad & $5(25 \%)$ & $3(15 \%)$ \\
$16-30$ años & $2(10 \%)$ & $7(35 \%)$ \\
$31-55$ años & $2(10 \%)$ & $1(5 \%)$ \\
$56-64$ años & & \\
\hline Escolaridad & $3(15 \%)$ & $2(10 \%)$ \\
$0-3$ años & $4(20 \%)$ & $5(25 \%)$ \\
$4-9$ años & $2(10 \%)$ & $4(20 \%)$ \\
$10-22$ ańos & & \\
\hline Estado civil & $3(15 \%)$ & $4(20 \%)$ \\
Soltero(a) & $2(10 \%)$ & $5(25 \%)$ \\
Casado(a) & $4(20 \%)$ & $2(10 \%)$ \\
Unión Libre & & \\
\hline Ocupación & $6(35 \%)$ \\
Labores del hogar & $3(15 \%)$ & $2(10 \%)$ \\
Artesanías & 0 & $2(10 \%)$ \\
Agricultura & $3(15 \%)$ & $2(10 \%)$ \\
\hline Procedencia & $4(20 \%)$ & 0 \\
Pasto & $2(10 \%)$ & \\
Putumayo & & \\
Municipios de Nariño & & \\
Municipios fuera de Nariño & & \\
\hline & & \\
\hline
\end{tabular}

traumático y a la presencia de síntomas persistentes de hipo-activación respectivamente. Su punto de corte es equivalente a 40 , por lo cual, puntajes superiores a este valor se relacionan con la existencia de TEPT (Bados, 2005). La escala cuenta con propiedades psicométricas 
apropiadas para la evaluación de este trastorno, con un $\alpha$ de .99 como lo sugieren Villafañe et al. (2003), quienes a su vez retoman a sus creadores Davidson et al. (1997), evaluando distintas muestras de pacientes, en total 353 víctimas de distintos traumas (violación, veteranos de guerra, víctimas del Huracán Andrew y supervivientes de diversos acontecimientos traumáticos que participaban en un ensayo clínico).

La evaluación de los procesos de Atención y Memoria se realizó a partir de los resultados del NEUROPSI (Ostrosky, Gómez, Ardila, Rosselli, Pineda \& Matute, 2012). Este instrumento evalúa la atención sostenida, selectiva, el control atencional, así como los procesos de memoria de trabajo a corto y largo plazo. Igualmente, toma en cuenta la orientación a partir del nivel de conciencia y el estado general de activación de la persona. Esta batería permite detectar trastornos cognitivos en población entre 6 y 85 años que puede ser analfabeta o con baja escolaridad, debido a que los reactivos que la conforman son sencillos y cortos, lo cual representa una ventaja para su aplicación (Ostrosky et al., 2012). Los datos normativos de esta batería se obtuvieron con su aplicación a un total de 950 individuos hispanohablantes sanos entre 6 y 85 años, divididos en rangos de edad y estratificados por nivel educativo.

\section{Procedimiento}

En primera instancia se llevó a cabo el proceso de selección de los 20 participantes en el presente estudio, con ellos se diligenció el consentimiento informado, en el que se detalla sus derechos y aspectos generales de la investigación. Una vez seleccionada la muestra, se procedió a formar el grupo A y grupo B, por medio de la aplicación de la escala DTS, cuya puntuación permite definir personas en las cuales se identifica o no sintomatología de TEPT.

Teniendo en cuenta que el punto de corte de esta escala es de 40, $y$ por tanto que en valores superiores se indica presencia de sintomatología de TEPT, se conformó el grupo A con un total de 9 personas con TEPT, quienes obtuvieron puntajes superiores a esta cifra; mientras que, el grupo B, fue conformado por 11 sujetos sin TEPT, con 
puntajes inferiores en el instrumento. Una vez distribuidos los grupos, se administró el protocolo de evaluación de NEUROPSI (Atención y Memoria), mediante el cual se determina un índice cuantitativo por separado de los procesos atencional y mnémico y un índice del puntaje total en conjunto del circuito atención - memoria. Al ser concluido el proceso de evaluación se realizó la calificación y puntuación de cada uno de los protocolos de forma individual, se tabularon los resultados y se calcularon porcentajes estadísticos a nivel general, en la finalidad de lograr interpretar el rendimiento de atención y memoria de los sujetos objeto del estudio y contrastarlos entre el grupo A (con TEPT) y el grupo B (sin TEPT), para así, poder interpretar la información recolectada durante el proceso y retroalimentarla con coincidencias o disonancias encontradas al ser relacionadas con investigaciones y trabajos similares desarrollados años atrás.

\section{Resultados}

Se presentan los resultados obtenidos por medio de la aplicación de la batería NEUROPSI a los participantes del estudio. En primer lugar, se describe de forma aislada el rendimiento del grupo A (con TEPT) y del grupo B (sin TEPT), frente a los procesos de atención y memoria respectivamente; luego, se indican los resultados que ambos grupos obtuvieron en la puntuación global del circuito atención - memoria. Asimismo, se enseńa un análisis por componentes que posibilita una mejor comprensión del perfil neuropsicológico de la muestra, en el cual se detalla específicamente el rendimiento de los evaluados en cada una de las subpruebas que corresponden a los procesos atencionales y mnémicos, e identifica en cuál de ellas existe mayor implicación.

Al comparar puntuaciones en el desempeño de atención entre los grupos A y B, se evidenció una relación de 4 a 2 sujetos con alteración en este proceso cognitivo, por lo cual se demuestra que existe mayor implicación en sujetos del grupo A (con TEPT), que puede asociarse a la presencia de este trastorno, como se observa en la tabla 2. 


\section{Tabla 2}

Nivel de desempeño cognitivo en el proceso atencional

\begin{tabular}{lcc}
\hline $\begin{array}{l}\text { Nivel de desempeńo cognitivo } \\
\text { en el proceso atencional }\end{array}$ & $\begin{array}{c}\text { Grupo A } \\
\mathbf{9 ( 4 5 \% )}\end{array}$ & $\begin{array}{c}\text { Grupo B } \\
\mathbf{1 1}(55 \%)\end{array}$ \\
\hline Normal alto & 0 & $2(10 \%)$ \\
Normal & $5(25 \%)$ & $6(30 \%)$ \\
Alteración leve/moderada & $4(20 \%)$ & $2(10 \%)$ \\
Alteración severa & 0 & $1(5 \%)$ \\
\hline
\end{tabular}

A nivel de memoria se visualiza que tanto el grupo A como el grupo B, tuvieron un rendimiento normal para la ejecución de las tareas; sin embargo, se destaca un mejor rendimiento en sujetos con TEPT, demostrando que no existe una relación directa entre TEPT y el proceso mnémico (ver Tabla 3).

\section{Tabla 3}

Nivel de desempeño cognitivo en el proceso de memoria

\begin{tabular}{lcc}
\hline $\begin{array}{l}\text { Nivel de desempeño cognitivo } \\
\text { en el proceso de memoria }\end{array}$ & $\begin{array}{c}\text { Grupo A } \\
\mathbf{9 ( 4 5 \% )}\end{array}$ & $\begin{array}{c}\text { Grupo B } \\
\mathbf{1 1}(55 \%)\end{array}$ \\
\hline Normal alto & $1(5 \%)$ & $1(5 \%)$ \\
Normal & $5(25 \%)$ & $6(30 \%)$ \\
Alteración leve/ moderada & $2(10 \%)$ & $4(20 \%)$ \\
Alteración severa & $1(5 \%)$ & 0 \\
\hline
\end{tabular}

En lo que refiere al desempeño global del circuito atenciónmemoria, se observa una alteración leve con mayor incidencia en sujetos del grupo B (sin TEPT), aunque existen casos de baja representatividad del grupo A (con TEPT) que demuestran alteración leve y severa frente a la interacción de ambos procesos (ver Tabla 4).

El análisis por componentes para conocer cada uno de los puntajes obtenidos de forma independiente en las subpruebas de atención y de memoria se describe a fin de especificar en cuál de estas se visualiza un mayor compromiso en uno u otro grupo. 
Perfil neuropsicológico de atención y memoria en víctimas del conflicto armado colombiano /Luna et al.

\section{Tabla 4}

Nivel de desempeño cognitivo en los procesos de atención y memoria

\begin{tabular}{lcc}
\hline $\begin{array}{l}\text { Nivel desempeńo cognitivo } \\
\text { procesos de atención y memoria }\end{array}$ & $\begin{array}{c}\text { Grupo A } \\
\mathbf{9 ( 4 5 \% )}\end{array}$ & $\begin{array}{c}\text { Grupo B } \\
11(55 \%)\end{array}$ \\
\hline Normal alto & $3(15 \%)$ & $2(10 \%)$ \\
Normal & $3(15 \%)$ & $4(20 \%)$ \\
Alteración leve/moderada & $2(10 \%)$ & $5(25 \%)$ \\
Alteración severa & $1(5 \%)$ & 0 \\
\hline
\end{tabular}

De este modo, frente a la interpretación del rendimiento en tareas relacionadas al proceso atencional, se puede decir que existe una alteración moderada en dígitos en progresión por parte del grupo $\mathrm{B}$ (sin TEPT); mientras que en detección de dígitos a pesar de que ambos grupos obtuvieron un puntaje de normalidad, sujetos sin TEPT se desempeñaron de mejor manera en comparación a los del grupo con TEPT (ver Tabla 5).

\section{Tabla 5}

Componentes del proceso atencional

\begin{tabular}{|c|c|c|c|c|c|c|c|c|}
\hline \multirow{3}{*}{$\begin{array}{l}\text { Componentes } \\
\text { del proceso } \\
\text { atencional }\end{array}$} & \multicolumn{8}{|c|}{ Nivel de desempeño } \\
\hline & \multicolumn{2}{|c|}{ Alt. severa } & \multicolumn{2}{|c|}{$\begin{array}{l}\text { Alt. leve/ } \\
\text { moderada }\end{array}$} & \multicolumn{2}{|c|}{ Normal } & \multicolumn{2}{|c|}{ Normal alto } \\
\hline & Grupo & Grupo B & Grupo A & Grupo B & Grupo A & Grupo B & Grupo A & Grupo B \\
\hline $\begin{array}{l}\text { Dígitos en } \\
\text { progresión }\end{array}$ & 0 & $\begin{array}{c}3 \\
(15 \%)\end{array}$ & 0 & $\begin{array}{c}8 \\
(40 \%)\end{array}$ & $\begin{array}{c}8 \\
(40 \%)\end{array}$ & 0 & $1(5 \%)$ & 0 \\
\hline $\begin{array}{l}\text { Cubos } \\
\text { progresión }\end{array}$ & 0 & 0 & $\begin{array}{c}3 \\
(15 \%)\end{array}$ & $\begin{array}{c}2 \\
(10 \%)\end{array}$ & $\begin{array}{c}3 \\
(15 \%)\end{array}$ & $\begin{array}{c}8 \\
(40 \%)\end{array}$ & $\begin{array}{c}3 \\
(15 \%)\end{array}$ & $\begin{array}{c}1 \\
(5 \%)\end{array}$ \\
\hline $\begin{array}{l}\text { Detección } \\
\text { visual }\end{array}$ & 0 & $\begin{array}{c}1 \\
(5 \%)\end{array}$ & $\begin{array}{c}1 \\
(5 \%)\end{array}$ & $\begin{array}{c}1 \\
(5 \%)\end{array}$ & $\begin{array}{c}6 \\
(30 \%)\end{array}$ & $\begin{array}{c}5 \\
(25 \%)\end{array}$ & $\begin{array}{c}2 \\
(10 \%)\end{array}$ & $\begin{array}{c}4 \\
(20 \%)\end{array}$ \\
\hline $\begin{array}{l}\text { Detección de } \\
\text { dígitos }\end{array}$ & 0 & 0 & $\begin{array}{c}1 \\
(5 \%)\end{array}$ & $\begin{array}{c}1 \\
(5 \%)\end{array}$ & $\begin{array}{c}6 \\
(30 \%)\end{array}$ & $\begin{array}{c}10 \\
(50 \%)\end{array}$ & $\begin{array}{c}2 \\
(10 \%)\end{array}$ & 0 \\
\hline $\begin{array}{l}\text { Series } \\
\text { sucesivas }\end{array}$ & 0 & 0 & 0 & $\begin{array}{c}1 \\
(5 \%)\end{array}$ & $\begin{array}{c}7 \\
(35 \%)\end{array}$ & $\begin{array}{c}5 \\
(25 \%)\end{array}$ & $\begin{array}{c}2 \\
(10 \%)\end{array}$ & $\begin{array}{c}5 \\
(25 \%)\end{array}$ \\
\hline
\end{tabular}


A continuación se presentan los componentes del proceso mnémico, por nivel de desempeño (ver Tabla 6).

Tabla 6

Componentes del proceso mnémico por nivel de desempeño

\begin{tabular}{|c|c|c|c|c|c|c|c|c|}
\hline \multirow{3}{*}{$\begin{array}{l}\text { Componentes } \\
\text { del proceso } \\
\text { mnémico }\end{array}$} & \multicolumn{8}{|c|}{ Nivel de desempeńo } \\
\hline & \multicolumn{2}{|c|}{ Severo } & \multicolumn{2}{|c|}{ Leve/Moderado } & \multicolumn{2}{|c|}{ Normal } & \multicolumn{2}{|c|}{ Normal Alto } \\
\hline & $\underset{A}{\text { Grupo }}$ & $\underset{\mathrm{B}}{\text { Grupo }}$ & $\underset{\text { A }}{\text { Grupo }}$ & $\underset{\mathrm{B}}{\text { Grupo }}$ & $\underset{\mathrm{A}}{\text { Grupo }}$ & $\underset{\mathrm{B}}{\text { Grupo }}$ & $\underset{\mathrm{A}}{\text { Grupo }}$ & $\underset{\mathrm{B}}{\text { Grupo }}$ \\
\hline $\begin{array}{l}\text { Dígitos en } \\
\text { regresión }\end{array}$ & 0 & $\begin{array}{c}1 \\
(5 \%)\end{array}$ & $\begin{array}{c}2 \\
(10 \%)\end{array}$ & 0 & $\begin{array}{c}6 \\
(30 \%)\end{array}$ & $\begin{array}{c}9 \\
(45 \%)\end{array}$ & $\begin{array}{c}1 \\
(5 \%)\end{array}$ & $\begin{array}{c}1 \\
(5 \%)\end{array}$ \\
\hline Cubos regresión & $\begin{array}{c}2 \\
(10 \%)\end{array}$ & $\begin{array}{c}1 \\
(5 \%)\end{array}$ & 0 & $\begin{array}{c}3 \\
(15 \%)\end{array}$ & $\begin{array}{c}6 \\
(30 \%)\end{array}$ & $\begin{array}{c}7 \\
(35 \%)\end{array}$ & $\begin{array}{c}1 \\
(5 \%)\end{array}$ & 0 \\
\hline $\begin{array}{l}\text { Curva de } \\
\text { memoria (Cod) }\end{array}$ & 0 & 0 & $\begin{array}{c}3 \\
(15 \%)\end{array}$ & $\begin{array}{c}5 \\
(25 \%)\end{array}$ & $\begin{array}{c}5 \\
(25 \%)\end{array}$ & $\begin{array}{c}6 \\
(30 \%)\end{array}$ & $\begin{array}{c}1 \\
(5 \%)\end{array}$ & 0 \\
\hline $\begin{array}{l}\text { Pares asociados } \\
\text { (Cod) }\end{array}$ & 0 & 0 & $\begin{array}{c}2 \\
(10 \%)\end{array}$ & $\begin{array}{c}1 \\
(5 \%)\end{array}$ & $\begin{array}{c}5 \\
(25 \%)\end{array}$ & $\begin{array}{c}8 \\
(40 \%)\end{array}$ & $\begin{array}{c}2 \\
(10 \%)\end{array}$ & $\begin{array}{c}2 \\
(10 \%)\end{array}$ \\
\hline $\begin{array}{l}\text { Memoria lógica } \\
\text { historias (Cod) }\end{array}$ & $\begin{array}{c}1 \\
(5 \%)\end{array}$ & 0 & $\begin{array}{c}1 \\
(5 \%)\end{array}$ & 0 & $\begin{array}{c}5 \\
(25 \%)\end{array}$ & $\begin{array}{c}11 \\
(55 \%)\end{array}$ & $\begin{array}{c}2 \\
(10 \%)\end{array}$ & 0 \\
\hline $\begin{array}{l}\text { Figura semi- } \\
\text { compleja (Cod) }\end{array}$ & 0 & $\begin{array}{c}1 \\
(5 \%)\end{array}$ & 0 & $\begin{array}{c}1 \\
(5 \%)\end{array}$ & 0 & $\begin{array}{c}8 \\
(40 \%)\end{array}$ & $\begin{array}{c}1 \\
(5 \%)\end{array}$ & $\begin{array}{c}1 \\
(5 \%)\end{array}$ \\
\hline Caras (Cod) & $\begin{array}{c}2 \\
(10 \%)\end{array}$ & $\begin{array}{c}1 \\
(5 \%)\end{array}$ & $\begin{array}{c}3 \\
(15 \%)\end{array}$ & 0 & $\begin{array}{c}4 \\
(20 \%)\end{array}$ & $\begin{array}{c}10 \\
(50 \%)\end{array}$ & 0 & 0 \\
\hline $\begin{array}{l}\text { Memoria verbal } \\
\text { espontanea (Ev) }\end{array}$ & $\begin{array}{c}1 \\
(5 \%)\end{array}$ & 0 & $1(5 \%)$ & 0 & $\begin{array}{c}5 \\
(25 \%)\end{array}$ & $\begin{array}{c}11 \\
(55 \%)\end{array}$ & $\begin{array}{c}2 \\
(10 \%)\end{array}$ & 0 \\
\hline $\begin{array}{l}\text { Memoria verbal } \\
\text { claves (Ev) }\end{array}$ & $\begin{array}{c}2 \\
(10 \%)\end{array}$ & 0 & $1(5 \%)$ & 0 & $\begin{array}{c}4 \\
(20 \%)\end{array}$ & $\begin{array}{c}11 \\
(55 \%)\end{array}$ & $\begin{array}{c}2 \\
(10 \%)\end{array}$ & 0 \\
\hline $\begin{array}{l}\text { Memoria verbal } \\
\text { reconocimiento } \\
\text { (Ev) }\end{array}$ & 0 & $\begin{array}{c}1 \\
(5 \%)\end{array}$ & $\begin{array}{c}1 \\
(5 \%)\end{array}$ & $\begin{array}{c}1 \\
(5 \%)\end{array}$ & $\begin{array}{c}7 \\
(35 \%)\end{array}$ & $\begin{array}{c}8 \\
(40 \%)\end{array}$ & $\begin{array}{c}1 \\
(5 \%)\end{array}$ & $\begin{array}{c}1 \\
(5 \%)\end{array}$ \\
\hline $\begin{array}{l}\text { Pares asociados } \\
\text { (Ev) }\end{array}$ & 0 & 0 & $\begin{array}{c}2 \\
(10 \%)\end{array}$ & $1(5 \%)$ & $\begin{array}{c}3 \\
(15 \%)\end{array}$ & $\begin{array}{c}9 \\
(45 \%)\end{array}$ & $\begin{array}{c}4 \\
(20 \%)\end{array}$ & $\begin{array}{c}1 \\
(5 \%)\end{array}$ \\
\hline $\begin{array}{l}\text { Memoria lógica } \\
\text { historias (Ev) }\end{array}$ & $\begin{array}{c}1 \\
(5 \%)\end{array}$ & 0 & $\begin{array}{c}1 \\
(5 \%)\end{array}$ & $\begin{array}{c}2 \\
(10 \%)\end{array}$ & $\begin{array}{c}5 \\
(25 \%)\end{array}$ & $\begin{array}{c}7 \\
(35 \%)\end{array}$ & $\begin{array}{c}2 \\
(10 \%)\end{array}$ & $\begin{array}{c}2 \\
(10 \%)\end{array}$ \\
\hline $\begin{array}{l}\text { Figura semi- } \\
\text { compleja (Ev) }\end{array}$ & 0 & 0 & 0 & $\begin{array}{c}2 \\
(10 \%)\end{array}$ & $\begin{array}{c}7 \\
(35 \%)\end{array}$ & $\begin{array}{c}6 \\
(30 \%)\end{array}$ & $\begin{array}{c}2 \\
(10 \%)\end{array}$ & $\begin{array}{c}3 \\
(15 \%)\end{array}$ \\
\hline $\begin{array}{l}\text { Reconocimiento } \\
\text { de caras (Ev) }\end{array}$ & 0 & 0 & $\begin{array}{c}1 \\
(5 \%)\end{array}$ & 0 & $\begin{array}{c}7 \\
(35 \%)\end{array}$ & $\begin{array}{c}11 \\
(55 \%)\end{array}$ & $\begin{array}{c}1 \\
(5 \%)\end{array}$ & 0 \\
\hline
\end{tabular}


Perfil neuropsicológico de atención y memoria en víctimas del conflicto armado colombiano /Luna et al.

\section{Discusión}

El conflicto armado colombiano y el desplazamiento forzado como consecuencia de este, ha afectado a un gran número de personas con el pasar del tiempo, a través de los más de 50 años de duración que ha tenido en Colombia; en el que se cuentan diversidad de hechos de violencia. Indican Vasterling, Duke, Brailey, Constans, Allain \& Sutker (2002) y Calderón y Barrera (2012) que entre sus principales secuelas se encuentra el TEPT, trastorno que no solo ha sido relacionado con la sintomatología que lo acompaña, sino también por la aparición de déficits en atención y memoria de quienes lo padecen.

Los resultados de la presente investigación muestran que, sujetos en los cuales se evidencia TEPT tienen un rendimiento inferior frente al proceso atencional, en comparación a aquellos sin TEPT; en este sentido, Ostrosky, Gómez, Ardila, Rosselli, Pineda \& Matute (2012) consideran que una alteración en atención puede reflejarse en dificultades de focalización y discriminación de estímulos para la realización de una tarea; tal cual se evidenció durante el proceso de evaluación en sujetos pertenecientes al grupo A (con TEPT). Así mismo, autores como Jurado et al. (2007) mencionan las causas neuro-anatómicas que sustentan estos déficits, especificadas por una alteración en sujetos con TEPT en áreas con receptores noradrenérgicos, como la amígdala e hipocampo, que puede traducirse en una disfunción de la eficacia cognitiva a nivel de atención.

Concuerdan a su vez trabajos como el de Seijas (2012 y 2013), en los cuales se evidencia la relación del TEPT con dificultades en atención de tipo focalizada, sostenida, alternante y selectiva, a causa de una implicación estructural en la corteza frontal, que de igual modo se asocia con una alteración en el procesamiento de información; en concordancia con autores como Bernate-Navarro, Baquero-Vargas y Soto-Pérez (2009) quienes hablan acerca de una afectación en la ejecución de atención selectiva y alternante, relacionados a velocidad de procesamiento de información.

Por otra parte, en el proceso mnémico no existen diferencias estadísticamente significativas entre los dos grupos, sin embargo, se visualizan 
algunos casos identificados de personas con TEPT en los cuales existe una alteración a nivel de memoria. $\mathrm{Al}$ respecto, investigaciones previas como la de Jurado et al (2007) determinan que sujetos con TEPT en comparación con un grupo de sujetos sin TEPT muestran un patrón diferente en las fases de consolidación y recuperación de la información en lo que refiere a aprendizaje verbal; mientras que en memoria visual el rendimiento es similar en los dos grupos. Lo anterior, está en concordancia con el presente trabajo, en tanto, si bien los resultados obtenidos manifiestan una implicación a nivel de memoria verbal, no determinan que exista una afectación cuando la tarea involucra estímulos visuales (Figura Compleja de Rey), pues el rendimiento es normal en los grupos A y B para esta prueba.

Por lo tanto, se puede evidenciar en este caso que, el TEPT no es una variable que pueda relacionarse directamente con la existencia de implicaciones a nivel de memoria, pues, aunque existen casos de baja representatividad en donde es posible visualizar una alteración leve o severa en ambos grupos, los porcentajes determinan que hay una mayor afectación en los sujetos sin TEPT; por lo cual, se supone la influencia de otros elementos que expliquen este fenómeno. Tal como lo señala el estudio realizado por Neylan et al (2004), en los cuales no se encontraron diferencias significativas del rendimiento de grupos con y sin TEPT y cuyos autores justifican a causa de factores influyentes como nivel de educación, edad, diferencias en el volumen hipocampal y factores de exclusión muy estrictos en la conformación de la muestra.

Igualmente, estos hallazgos pueden entenderse desde diferentes autores como Ostrosky-Solís, Ardila, Rosselli, López-Arango \& UrielMendoza (1998), Ardila, Ostrosky-Solis, Rosselli \& Gómez (2000), Gómez-Pérez \& Ostrosky-Solís (2006) y Castillo, Gómez y OstroskySolis (2009), quienes comprenden la influencia de factores de tipo sociodemográfico frente al rendimiento cognitivo de los participantes desde el nivel de estudios; o en la misma línea, también Ardila \& Rosselli (1996) y Mejía (2013) se refieren al -gradiente socioeconómico y gradiente de integridad neurológica-, haciendo referencia a factores de riesgo relacionados con las características de niveles socioeconómicos que en cierto modo afectan las funciones cognitivas del sujeto. 
Perfil neuropsicológico de atención y memoria en víctimas del conflicto armado colombiano /Luna et al.

\section{Conclusiones}

A partir de la evaluación neuropsicológica de atención y memoria desarrollada con los participantes del proyecto y los resultados encontrados, se deduce que la presencia del TEPT incide directamente a nivel atencional, manifestándose afectaciones cognitivas en este proceso en sujetos que padecen del trastorno, pues los hallazgos del presente estudio demuestran que personas del grupo A (con TEPT) obtuvieron un rendimiento inferior en la ejecución de tareas de carácter atencional.

En cambio, a nivel de memoria existe mayor alteración en sujetos del grupo B (sin TEPT), por lo cual no se puede establecer que el TEPT influye directamente el proceso mnémico; en tanto; es pertinente considerar una serie de factores externos como lo son aspectos sociodemográficos de: nivel de escolaridad, elementos socioeconómicos y la situación de desplazamiento, que a su vez influyen en el desempeño cognitivo de la persona. Del mismo modo sucede este fenómeno con el puntaje global del circuito atención - memoria, donde tampoco se demuestra afectación en los sujetos con TEPT, y por lo cual también se debe tener en cuenta los factores ya mencionados.

El presente estudio cumple con el propósito con el cual fue planteado, pues partiendo de los datos recolectados se pudo obtener una comprensión más amplia frente a la población víctima de desplazamiento a causa del conflicto armado que sufre de TEPT y las dificultades que pueden generarse desde un ámbito neuropsicológico; en este caso, netamente de carácter atencional, con lo cual se espera que futuras investigaciones sean realizadas a fin de tener mayor conocimiento respecto al tema y que alienten a que se realicen ajustes frente a la atención integral que es prestada a las víctimas del conflicto. Para ello, además se nombran las limitaciones presentadas en la realización de la investigación, relacionadas principalmente con el tamaño y las características de la muestra de trabajo, pues debe tenerse en cuenta que al trabajar con un mayor número de personas entre las cuales exista más homogeneidad frente a variables sociodemográficas, es posible tener un análisis más completo y detallado. 


\section{Referencias}

Ardila, A., Ostrosky-Solis, F., Rosselli, M. \& Gómez, C. (2000). Age related cognitive decline during normal aging: The complex effect of education. Archives of Clinical Neuropsychology, 15(6), 495-513. Ardila, A \& Rosselli, M. (2009). Soft neurological signs in children: A normative study. Developmental Neuropsychology, 12(1), 181200. http://dx.doi.org/10.1080/87565649609540645

Bados, A. (2005). Trastorno por Estrés Postraumático. Barcelona: universidad de Barcelona. Recuperado de http://diposit.ub.edu/ dspace/bitstream/2445/356/1/117.pdf

Bernate-Navarro, M., Baquero-Vargas, M. \& Soto-Pérez, F. (2009). Diferencias en los procesos de atención y memoria en niños con y sin estrés postraumático. Cuad. Neuropsicol, 3(1), 104-115.

Calderón, D. \& Barrera, M. (2012). Exploración neuropsicológica de la atención y la memoria en niños y adolescentes víctimas de la violencia en Colombia: estudio preliminar. Revista CES Psicologia, 5(1), 39-48.

Barrera, M. \& Calderón, L. (2016). Perfil neuropsicológico del trastorno por estrés postraumático agudo en una muestra de personas, víctimas de un atentado con carro-bomba en Colombia: estudio descriptivo. Archivos de Medicina, 16(1), 89-97.

Castillo, G., Gomez, E. \& Ostrosky, F. (2009). Relación entre las funciones cognitivas y el nivel de rendimiento académico en niños. Revista Neuropsicología, Neuropsiquiatría y Neurociencias, 9(1), 41-54.

Coelho, L. \& Costa, J. (2010). Bases neurobiológicas del estrés postraumático. Anales de Psicología, 26(1), 1-10.

Espitia, J. (2015) Los hechos victimizantes a nivel departamental (1985-2014). En Seminario virtual viva la ciudadanía. Edición 442. Recuperado de http://viva.org.co/cajavirtual/svc0442/ pdfs/Articulo160_442.pdf

Gálvez, J. F. (2005). Trastorno por Estrés y sus repercusiones neuropsicoendocrinologicas. Asociación Colombiana de Psiquiatría, 34(1), 77-100. 
Gómez-Pérez, E. \& Ostrosky-Solís, F. (2006). Attention and memory evaluation across the life span: heterogeneous effects of age and education. J Clin Exp Neuropsychol, 28(4), 477-494. https://doi. org/10.1080/13803390590949296

Hernández, I., Luna, J. \& Cadena, M. (2017). Cultura de paz: una construcción desde la educación. Revista Historia de la Educación Latinoamericana SHEL, 19(28), 149-172. https://doi. org/10.19053/01227238.5596

Hurtado, C. (2011). Alteraciones neuropsicológicas en el estrés postraumático. Universidad de Salamanca, Facultad de Psicología, Master Universitario en Neuropsicología Universidad de Salamanca. Recuperado de http://hdl.handle.net/10366/115856

Jurado, B., Taboada, D., García, B., Denia, R., Mingote, A. \& Fernández, G. (2007). Implicación del hipocampo y la amígdala en el rendimiento neuropsicológico de pacientes con trastorno por estrés postraumático. Mapfre Medicina, 18(1), 92-101.

Melamed, J. (2014). Del conflicto al posconflicto en el contexto colombiano. Revista Universidad de La Salle, 63, 57-73.

Mejía, M. (2013). Características cognitivas de niños y niñas en situación de desplazamiento residentes en Colombia. Maestría en Desarrollo Infantil, Facultad de Ciencias Sociales y Humanas. Universidad de Manizales.

Neylan, T., Lenoci, M., Rothlind, J., Metzler, T., Schuff, N., Du, A., Franklin, K., Weiss, D., Weiner, M. \& Marmar, C. (2004) Attention, learning, and memory in posttraumatic stress disorder. Journal of Traumatic Stress, 17(1), 41-46. https:// dx.doi.org/10.1023/B:JOTS.0000014675.75686.ee

Ostrosky, F., Gómez, E., Ardila, A., Rosselli, M., Pineda, D. \& Matute, E. (2012). Neuropsi. Atención y memoria. Protocolo de aplicación. $2^{a}$ edición. México: Manual Moderno.

Ostrosky-Solis, F., Ardila, A., Rosselli, M., Lopez-Arango, G. \& UrielMendoza, V. (1998). Neuropsychological test performance in illiterate subjects, Arch Clin Neuropsychol,13(7), 645-660.

Quintero, C. (2011). Memoria autobiográfica y semántica en el trastorno por estrés postraumático en desmovilizados individuales residentes 
en el municipio de Medellín. Magíster en Neuropsicología. Facultad de Psicología. Universidad San Buenaventura sede Medellín. Recuperado de http://bibliotecadigital.usbcali.edu.co/ bitstream/10819/281/1/Memoria_Autobiografica_Semantica_ Quintero_2011.pdf

Seijas, R. (2012). Aspectos neurobiológicos y neuropsicológicos del trastorno por estrés postraumático. C. Med. Psicosom, 104, 19-28.

Seijas, R. (2013). Trastorno por estrés postraumático y cerebro. Rev. Asoc. Esp. Neuropsiq, 33(119), 511-523. https://dx.doi. org/10.4321/S0211-57352013000300004

Vasterling, J., Duke, L., Brailey, K., Constans, J. Allain J. \& Sutker, P. (2002). Attention, learning, and memory performances and intellectual resources in Vietnam veterans: PTSD and no disorder comparisons. Neuropsychology, 16(1), 5-14. http:// dx.doi.org/10.1037/0894-4105.16.1.5

Villafañe, A., Milanesio, M., Marcellino, C. \& Amodei, C. (2003). La evaluación del trastorno por estrés postraumático: aproximación a las propiedades psicométricas de la Escala de Trauma de Davidson. Laboratorio de Evaluación Psicológica y Educativa, 3, 80-93.

Villarraga, A. (2013). Experiencias históricas recientes de reintegración de ex-combatientes en Colombia. Colombia Internacional, 77, 107-140. http://dx.doi.org/10.7440/colombiaint77.2013.05

Wilches, I. (2010). Lo que hemos aprendido sobre la atención a mujeres víctimas de violencia sexual en el conflicto armado colombiano. Revista de Estudios Sociales, 36, 86-94. https://dx.doi.org/ pdf/10.7440/res36.2010.07

Yaffe, K., Vittinghoff, E., Lindquist, K., Barnes, D., Covinsky, K., Neylan, T., Kluse, M. \& Marmar, C. (2010). Posttraumatic stress disorder and risk of dementia among US veterans. Arch Gen Psychiatry, 67(6), 608-613. https://dx.doi.org/10.1001/ archgenpsychiatry.2010.61

Recibido: 29 de noviembre, 2017

Revisado: 1 de marzo, 2018 Aceptado: 20 de mayo, 2018 